\title{
Cybrides virtuels : simuler l'influence du fond génétique mitochondrial sur le métabolisme
}

\author{
Margit Heiske, Lenka Tisseyre, Camille Fontes-Bouillet, Barbara DaCosta, Christophe Rocher, \\ Edda Klipp, Thierry Letellier
}

Les mitochondries sont les centrales énergétiques de nos cellules en fournissant $90 \%$ de l'énergie indispensable à la survie cellulaire (sous forme d'ATP). Le cœur du métabolisme énergétique mitochondrial est la phosphorylation oxydative (OXPHOS) où les complexes enzymatiques de la chaîne respiratoire établissent un gradient de protons qui est ensuite utilisé par l'ATP synthase pour la production d'ATP. Ce processus est en outre couplé à la production des dérivés réactifs de l'oxygène (ROS) qui jouent un rôle important dans la signalisation cellulaire mais qui sont également délétères pour les protéines, les lipides et l'ADN de la cellule. Les mitochondries sont aussi impliquées dans de nombreux autres processus cellulaires essentiels comme la thermogenèse, la signalisation calcique, l'apoptose, ainsi que la biosynthèse des acides aminés, des nucléotides et des acides gras. De par leur rôle central, une dysfonction mitochondriale est impliquée dans de nombreuses maladies (neuromusculaires, neurodégénératives, cancéreuses, etc.) ainsi que dans des processus physiologiques (vieillissement, adaptation humaine à de nouveaux environnements) [1, 2].

D'un point de vue phylogénétique, les mitochondries proviennent d'une fusion ancestrale entre une archéobactérie et une cellule primitive procaryote il y a plus de 1.5 milliards d'années [3] ; il en résulte qu'elles possèdent leur propre ADN (ADNmt). Si depuis les années 90, un grand nombre de mutations pathogènes sur l'ADNmt ont été impliquées dans des pathologies, récemment, des variations non pathologiques de l'ADN mitochondrial (polymorphismes) ont permis de définir des "haplogroupes mitochondriaux " qui regroupent des individus partageant les mêmes polymorphismes. En anthropologie, cette notion d'haplogroupe a très rapidement permis d'établir les relations parentales mitochondriales (arbre phylogénétique mitochondrial) ainsi que des scénarios de peuplement de l'espèce humaine. D'un point de vue clinique, les haplogroupes mitochondriaux se sont avérés être des facteurs de risque ou de protection vis-à-vis d'un grand nombre de pathologies comme les cytopathies mitochondriales, maladie de Parkinson, maladie d'Alzheimer, et cancer [4,5], cependant à ce jour, peu de bases biochimiques ou moléculaires claires ont été proposées pour expliquer cette influence.

Afin d'élucider ces mécanismes, nous avons utilisé une approche de biologie des systèmes (in silico) comprenant une partie théorique (construction du modèle mathématique) et expérimentale (afin de paramétrer et valider le modèle) (Figure 1). Cette approche devrait nous permettre de mieux comprendre l'influence des haplogroupes mitochondriaux sur le métabolisme énergétique dans le contexte de (1) mutations pathogènes, (2) facteurs environnementaux (climat, molécules nocives, rayonnement ionisant) et (3) nutrition.

L'approche théorique est basée sur notre modèle déterministe des OXPHOS $[6,7]$ qui permet une description précise de la fonction mitochondriale (flux respiratoire, production ATP) grâce aux équations de type Michaelis-Menten que nous avons développées pour décrire l'activité de chaque complexe OXPHOS sur un large intervalle de substrats, produits et gradient de protons. Récemment,



Figure 1

Approche de biologie des systèmes. 
nous avons étendu notre modèle OXPHOS par l'addition d'un module "ROS" (Figure 2). Ce module comprend des équations au niveau (1) biochimique pour décrire la production de ROS séparément pour chacun des complexes respiratoires I-III et (2) la dégradation enzymatique des ROS, basées sur le modèle de Gauthier et al. [8]. Dans un premier temps, ce modèle global (OXPHOS + ROS) a été paramétré et validé avec des données expérimentales de mitochondries de cour.

Nous avons étudié la relation entre la production de ROS et la production d'ATP dans différentes conditions (Figure 3A). Ainsi, selon nos simulations, la manière dont la cellule va moduler la production d'ATP va avoir de profondes implications sur la production de ROS. En effet, à l'état 3, une variation de substrats respiratoires impacterait plus la production de ROS qu'une variation du couplage ou de concentration en ADP.

D'un point de vue expérimental et afin d'étudier les effets des haplogroupes mitochondriaux sur le métabolisme énergétique, nous avons réalisé des lignées cellulaires transmitochondriales (cybrides: cellules ayant le même fond génétique nucléaire mais différent par leur fond génétique mitochondrial). Ces lignées cellulaires transmitochondriales sont en train d'être caractérisées au niveau biochimique et moléculaire (activité des complexes respiratoires, ATP synthase, état redox, etc.). En re-paramétrant notre modèle avec ces nouvelles données, nous comptons obtenir des "cybrides virtuels" qui prennent en compte des spécificités des haplogroupes au niveau local (activités enzymatiques) et permettent de simuler et prédire leur impact sur le système global d'OXPHOS (production d'ATP, taux respiratoire, potentiel membranaire, etc.) dans différentes conditions physiologiques.

Un résultat préliminaire que nous avons obtenu est présenté (Figure 3B). Nous avons simulé le cas de l'haplogroupe $\mathrm{J}$ qui présente une déficience en couplage au niveau du complexe III [4], en le comparant à une lignée ne présentant pas ce découplage. L'étude de la production de ROS par rapport à la production de l'ATP, montre qu'à l'état 3 (en présence d'ADP), nous observons pour l'haplogroupe $\mathrm{J}$ une production diminuée d'ATP, mais aussi de ROS, alors qu'à l'état 4 (absence d'ADP), la production des ROS est égale à la référence. Ainsi, pour l'haplogroupe J, cette légère diminution du couplage au niveau du complexe III peut être bénéfique car on produira moins de ROS. Cependant, lorsque les individus de l'haplogroupe $\mathrm{J}$ seront aussi porteurs d'une mutation pathogène touchant la chaîne respiratoire (entrainant une diminution de la production d'ATP) la légère diminution de production d'ATP déjà présente chez ces individus sains pourra aggraver le phénotype.

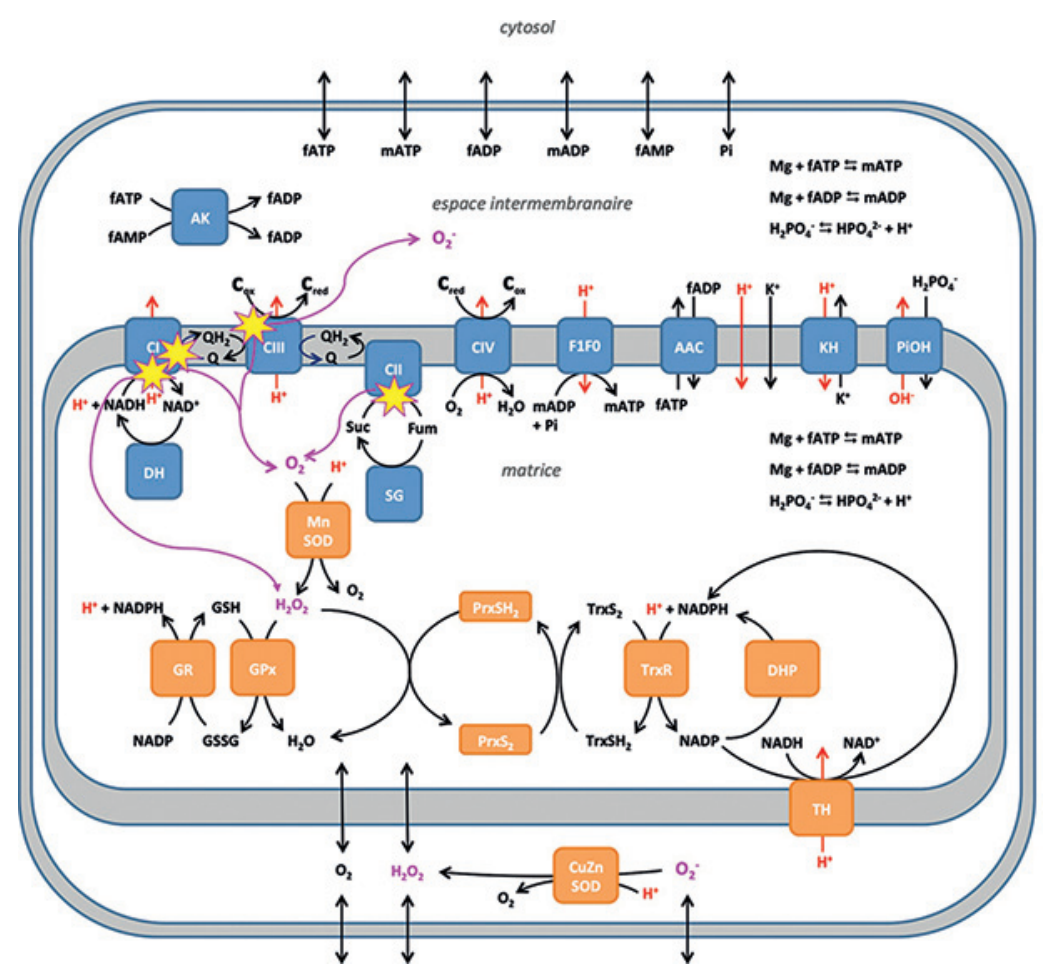

Figure 2

Représentation schématique du modèle avec les parties OXPHOS (bleu), production des ROS (jaune/rose) et de l'élimination des ROS (orange). 

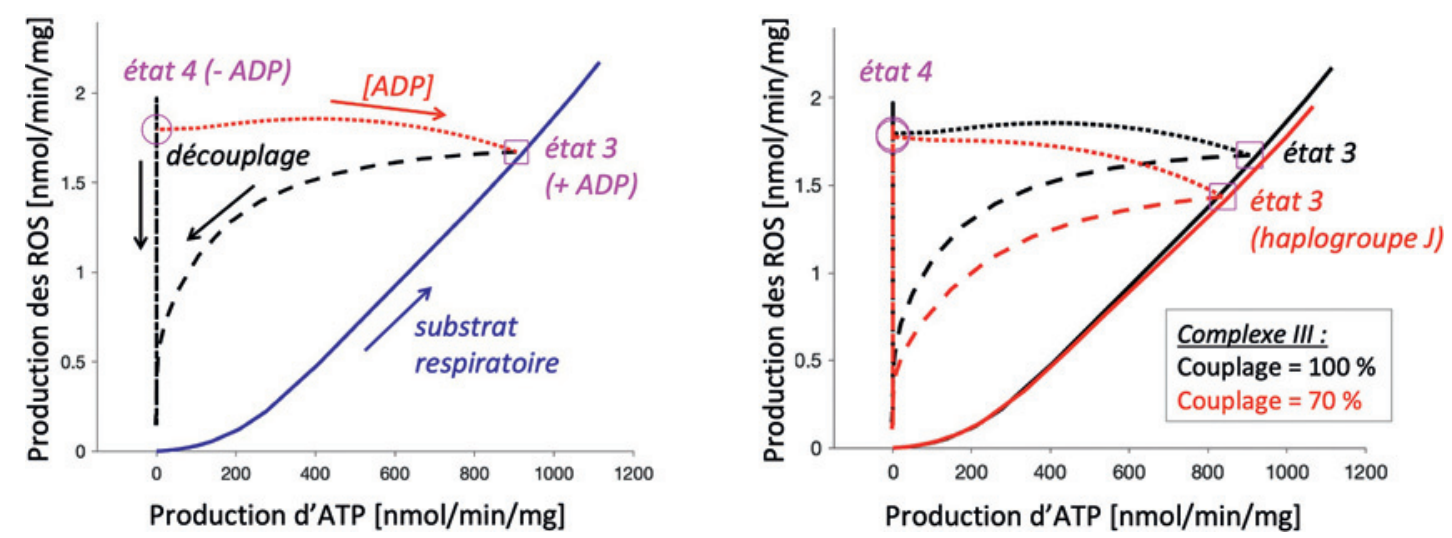

Figure 3

Simulation des relations entre production de ROS et production d'ATP sous différentes conditions.

3A: Mitochondrie de coeur : variation du substrat respiratoire (NADH) (bleu), variation de la concentration d'ADP (rouge) et variation du découplage (noir).

3B : Cybrides virtuels : En noir les simulations de référence (A), en rouge les simulations avec une déficience en couplage au niveau du complexe III.

À la vue de ces premiers résultats, les cybrides virtuels permettent donc d'analyser, prédire et tester des hypothèses sur le rôle des haplogroupes mitochondriaux au niveau du métabolisme énergétique, tant chez les individus sains que pathologiques. Notre modèle va aussi être étendu par un module "thermogénèse ", qui nous permettra d'étudier les équilibres de production d'ATP, ROS et chaleur indispensable pour une étude intégrée et complète du métabolisme mitochondrial. D'autre part, l'addition d'un module " apport de substrats respiratoires " (glucose, acides gras...), nous permettra de prendre aussi en compte les aspects de la nutrition. Cet outil de biologie des systèmes pourra aussi (1) aider au diagnostic notamment dans la prédiction de la sévérité et de la progression des maladies liées au métabolisme mitochondrial, et (2) améliorer et développer des approches thérapeutiques comme les restrictions alimentaires, en adaptant ces approches aux spécificités métaboliques des haplogroupes individuels.

Virtual cybrids: simulating the influence of the mitochondrial DNA background on the metabolism

\section{LIENS D'INTÉRÊT}

Les auteurs déclarent n'avoir aucun lien d'intérêt concernant les données publiées dans cet article.

\section{RÉFÉRENCES}

1. Wallace DC. A mitochondrial paradigm of metabolic and degenerative diseases, aging, and cancer: a dawn for evolutionary medicine. Annu Rev Genet 2005 ; 39 : 359-407.

2. Shapira AHV, Mitochondrial diseases. Lancet 2012; 379 1825-34.

3. Gray MW, Burger G, Lang BF. Mitochondrial evolution. Science 1999 Mar 5 ; 283 (5407) : 1476-81.

4. Pierron D, Rocher C, Amati-Bonneau P, et al. New evidence of a mitochondrial genetic background paradox: impact of the $\mathrm{J}$ haplogroup on the A3243G mutation. BMC Med Genet 2008; $9: 41$.

5. Marom S, Friger M, Mishmar D. MtDNA meta-analysis reveals both phenotype specificity and allele heterogeneity: a model for differential association. Sci Rep 2017; $7: 43449$.

6. Heiske M, Letellier T, Klipp E. Comprehensive mathematical model of oxidative phosphorylation valid for physiological and pathological conditions. FEBS J 2017; 284 : 2802-28.

7. Heiske M Nazaret C, Mazat JP. Modeling the respiratory chain complexes with biothermokinetic equations - the case of complex I. Biochim Biophys Acta 2014 ; 1837 : 1707-16.

8. Gauthier LD, Greenstein JL, Cortassa S, O'Rourke B, Winslow RL. A computational model of reactive oxygen species and redox balance in cardiac mitochondria. Biophys $J 2013 ; 105$ : 1045-56.

\section{Retrouvez toutes les Actualités de la Myologie} sur les sites de:

la Société Française de Myologie www.sfmyologie.org

la filière de santé neuromusculaire FILNEMUS www.filnemus.fr sfm



Filnemus 